\title{
Revisiter notre projet éditorial
}

Faut-il se réjouir du succès que connaît aujourd'hui l'appel à l'interdisciplinarité dans tout programme de recherche et, pour ce qui nous concerne, dans tout projet de revue scientifique?

Fondée voilà 20 ans, Natures Sciences Sociétés a construit sa réputation sur une telle référence: « recherches et débats interdisciplinaires » peut-on lire sur sa couverture. Et, si l'on s'en tient aux appartenances disciplinaires de ses auteurs, force est de constater qu'elles puisent dans le vivier des sciences humaines et sociales certes, mais que, pour plus d'un tiers ( $36 \%$ dans les 6 dernières années), les signataires viennent des sciences du vivant, de l'ingénieur, de la statistique, etc. Les associations entre les grands domaines disciplinaires y sont nombreuses et justifient la référence à l'interdisciplinarité de la revue.

Dès l'origine, les promoteurs de NSS ont associé cet objectif de construction d'une pratique scientifique interdisciplinaire et en quelque sorte « indisciplinée » à deux autres exigences : s'interroger sur la place de la production des savoirs dans la société et articuler la recherche avec des moyens d'action. Ce triptyque forme un tout et instruit ce qu' on entend par projet interdisciplinaire, une forme de recherche où prévaut le décloisonnement, qu'il soit entre disciplines ou au sein même de son lieu d'exercice, la société.

Une telle ambition était plutôt marginale à l'époque, même si les grands programmes interdisciplinaires, en particulier dans leur prise en charge des questions d'environnement, avaient déjà vu le jour dès les années 1970 à l'initiative de la Délégation générale à la recherche scientifique et technique (DGRST). Cette ambition est aujourd'hui à ce point partagée qu'elle représente l'alpha et l'oméga de toute programmation s'inscrivant dans l'excellence. Cela nous réjouit certes, mais cela nous interpelle aussi.

Ceux qui ont fait une véritable expérience interdisciplinaire savent que, de fait, une telle dynamique au sein d'un collectif de chercheurs est, par nature, non programmable. Le volontarisme dont font preuve les institutions est louable mais insuffisant pour impulser un «art de vivre ensemble » qui est au cœur de la pratique interdisciplinaire et sa condition première. Il peut même avoir un effet inverse en donnant le sentiment qu'une fois la diversité disciplinaire constituée, le contrat est rempli alors que tout reste à faire pour relever le défi, théorique et méthodologique, de la démarche interdisciplinaire. En d'autres termes, l'usage abusif du terme d'interdisciplinarité tend à en banaliser l'enjeu scientifique.

La conséquence immédiate de cette banalisation est une forme de brouillage de la position de NSS dans le champ éditorial où les revues affichant comme thématique première les questions environnementales se sont multipliées. Ce paysage est également brouillé par la diversification des supports qui ne se limitent plus à la version papier, consultable dans son ensemble. La généralisation du support électronique gomme les différentes formes d'expression (articles, textes d'opinion...), rendant obsolète la cohérence recherchée dans une ligne éditoriale. Or c'est cette cohérence des numéros, avec ses différentes rubriques complémentaires, qui mobilise les efforts du comité éditorial et qui constitue un des atouts originaux de NSS.

Par ailleurs, l'imposition des «normes de l'excellence", avec son cortège de validation par la course effrénée, d'un côté, au projet de recherche où l'innovation et le risque n'offrent pas les meilleures garanties d'accès, et, de l'autre côté, aux articles, de préférence en anglais publiés dans des revues avec facteur d'impact, modifie la pratique de recherche, tout au moins la façon d'exercer le métier sur le plan stratégique auquel renvoie l'acte de publier. La dimension collective du métier de chercheur n'a jamais été aussi prégnante dans ses conditions d'exercice, mais elle occulte de fait une individualisation croissante de la pratique de recherche, ce qui ne peut inciter à assumer les importants « coûts de transaction » que génère le choix interdisciplinaire. Ces normes peuvent éloigner de NSS les «jeunes chercheurs », contraints au sein du système qu'ils rejoignent 
de tenir compte des critères d'évaluation adoptés par leurs institutions.

Dans un tel contexte où la référence interdisciplinaire se banalise et où les questions environnementales suscitent une production éditoriale en tous genres, la question se pose de notre positionnement spécifique et du point de vue original que la revue veut faire valoir. Deux éléments nous apparaissent devoir être au cœur de notre engagement éditorial. Tout d'abord, il s'agit de continuer à promouvoir les débats et recherches interdisciplinaires pour en faire le creuset de pratiques permettant d'atteindre un haut niveau de recherche, non pas au nom d'une excellence qui se traduirait dans une concurrence accrue entre équipes et chercheurs, mais au nom d'une autre conception de l'excellence - ainsi que le recommande le comité d'éthique du CNRS (Comets $\left.{ }^{1}\right)-$ qui serait l'illustration d'une éthique collaborative et de valeurs partagées. Il s'agit en second lieu de se concentrer sur ce qui, au même titre que l'enjeu interdisciplinaire voilà 20 ans, interpelle la pratique scientifique aujourd'hui et sur ce qui constitue l'objectif central et le point fort de la revue : $\mathrm{s}^{\prime}$ interroger sur la place de la production des savoirs dans la société et encourager l'esprit critique face aux « évidences » et à la standardisation des approches et concepts.

Cette posture a une traduction directe dans le nouvel énoncé des objectifs de NSS que nous vous invitons à partager lorsqu'il affirme: "C'est en s'éloignant de points de vue strictement disciplinaires que Natures Sciences Sociétés interroge les "évidences" qui parcourent les démarches de recherche sur les questions environnementales, du développement durable à la résilience, de la gouvernance à l'approche écosystémique..., afin de partager une intelligibilité critique des rapports contemporains entre sciences et sociétés. »

Certes, NSS est identifiée comme une revue de référence sur le plan scientifique, exigeante sur le plan éditorial. Elle reste l'une des revues qui peut accueillir un texte dont la signature reflète la diversité des champs disciplinaires. Elle le doit à sa position pionnière par rapport à l'interdisciplinarité puisque c'est de cette exigence qu'elle est issue. Elle le doit également à la diversité de ses rubriques qui permet l'expression de postures réflexives, en particulier autour des rapports entre sciences et sociétés, qui sont autant de mises à distance avec la spécialisation croissante de l'expression disciplinaire. Elle le doit enfin à sa volonté de mettre en débat les grands enjeux contemporains et leurs implications sur la vie en société.

Jean-Paul Billaud, Catherine Aubertin et Bernard Hubert

1 La politique de l'excellence en recherche (co-saisine de la Présidence du CNRS et du Comité d'éthique), mai 2014, http://www.cnrs.fr/comets/IMG/pdf/avis_excellence.pdf. 